\title{
Nonlinear optical properties of ionic magnetic colloids in the femto- and millisecond time scales: change from convergent-to-divergent lens-type behaviors
}

\author{
Diogo Soga and Sarah Alves \\ Instituto de Física, Universidade de São Paulo, Caixa Postal 66318, 05315-970 São Paulo, São Paulo, Brazil \\ Alex Campos and Francisco Augusto Tourinho \\ Instituto de Química, Universidade de Brasília, Caixa Postal 04478, 70919-970 Brasília, Distrito Federal, Brazil \\ Jerôme Depeyrot \\ Instituto de Física, Universidade de Brasília, Caixa Postal 04455, 70919-970, Brasília, Distrito Federal, Brazil
}

Antonio Martins Figueiredo Neto

Instituto de Física, Universidade de São Paulo, Caixa Postal 66318, 05315-970 São Paulo, São Paulo, Brazil

Received May 26, 2006; revised August 8, 2006; accepted August 29, 2006;

posted September 6, 2006 (Doc. ID 71320); published December 20, 2006

\begin{abstract}
The Z-scan technique is used to measure the nonlinear refractive index $n_{2}$ and the nonlinear absorption coefficient $\beta$ of ionic magnetic colloids as a function of the nanoparticles' diameter $\left(D_{r x}\right)$ in the femto- and millisecond time-scale regimes. A change from a convergent-lens type (at a femtosecond time scale) to a divergent-lens type behavior (at a millisecond time scale) is observed. From the values of $n_{2}$ and $\beta$ measured in the femtosecond time-scale regime, the third-order susceptibility $\chi^{(3)}$ is calculated and is shown to depend on $D_{r x}$ : the smaller the $D_{r x}$, the bigger the $\chi^{(3)}$. A possible origin of the high values of $\chi^{(3)}$ found may be confining effects related to the physical nanoscale of the magnetic particles. (C) 2006 Optical Society of America

OCIS codes: $160.0160,190.4400,190.4720$.
\end{abstract}

\section{INTRODUCTION}

Different mechanisms can produce nonlinear effects in a material, in particular, changes in its refractive index. ${ }^{1}$ Among the mechanisms responsible for these changes, we have the electronic polarization, molecular orientation, electrostriction, saturated and atomic absorption, thermal effects, and photorefractive effects. Each of these mechanisms has a typical time response, ranging from $10^{-15} \mathrm{~s}$ (in the case of the electronic polarization) until $10^{-3} \mathrm{~s}$ (in the case of thermal effects). These mechanisms can be studied by measuring both the nonlinear refractive index $\left(n_{2}\right)$ and the nonlinear absorption coefficient $(\beta)$ of the material. In particular, in the case of electronic processes, the third-order nonlinear optical susceptibility $\left(\chi^{(3)}\right)$ can be calculated from these measured values of $n_{2}$ and $\beta$.

After the seminal work of Sheik-Bahae et al. ${ }^{2}$ proposing the Z-scan (ZS) technique, many works were published using it to investigate nonlinear properties of different materials. The ZS technique is used to measure both $n_{2}$ and $\beta$, and the great advantage of it lies on a very simple setup where a Gaussian profile laser beam is focused by a lens, causing a variation on the beam intensity along the $z$ direction. The sample, sensitive to the incident laser intensity, is moved through the focused beam path, and the transmitted beam intensity is detected by a photodetector placed behind an aperture. This technique can also be used to probe different nonlinear mechanisms, by adapting the experimental setup to the desired time scale of the phenomenon.

One of the most interesting complex fluids, due to its remarkable physical-chemical properties, is the ferrofluid. ${ }^{3}$ This complex fluid is a colloidal suspension of small magnetic particles (typical diameter of $\sim 10 \mathrm{~nm}$ ) dispersed in a liquid carrier (polar or nonpolar, depending on the particular characteristics of the magnetic particles's surface). The fluid is optically isotropic but, in the presence of a small magnetic field (e.g., $\sim 10^{2} \mathrm{G}$ ) shows field-induced birefringence and the formation of chains. Moreover, due to its high-magnetic susceptibility, flow properties are strongly dependent on the applied magnetic field. Among the different ferrofluids, the ionics (named here as IF) are particularly interesting ${ }^{4}$ : their magnetic particles are electrically charged, to avoid aggregation, and are dispersed in water. Besides their application in the doping of lyotropic liquid crystals, ${ }^{5}$ where 
their role is to improve the mesophases orientation in small magnetic fields, biocompatible ferrofluids were shown to be a powerful tool in medical therapy. ${ }^{6}$

The nanoscale of the ferrofluid particles, which can be pictured as a dot-type confined system, allows for the interesting study of the nonlinear response of this medium under optical stimuli. The measured third-order nonlinear optical susceptibility of transition metal oxides $(\alpha$ $-\mathrm{Fe}_{2} \mathrm{O}_{3}, \mathrm{TiO}_{2}, \gamma-\mathrm{Fe}_{2} \mathrm{O}_{3}$, and $\mathrm{Fe}_{3} \mathrm{O}_{4}$ ) in a form of thin films (i.e., a two-dimensional-type confined system) presented values as high as $10^{-12} \mathrm{esu}\left(\alpha-\mathrm{Fe}_{2} \mathrm{O}_{3}\right)$ (Ref. 7) to $10^{-10} \mathrm{esu}$ $\left(\mathrm{Fe}_{3} \mathrm{O}_{4}\right)^{8}$ These values are much bigger than those roughly predicted by using Miller's rule. ${ }^{9}$ For example, the predicted value of $\chi^{(3)}$ of $\mathrm{Fe}_{3} \mathrm{O}_{4}$ is of the order of 3 $\times 10^{-13}$ esu, 3 orders of magnitude smaller than the measured value. ZS experiments in the nanosecond time scale performed with $\mathrm{Fe}_{2} \mathrm{O}_{3}$-coated nanoparticles dispersed in toluene and hydrosol ${ }^{10}$ showed a nonlinear refractive index of the order of $-10^{-14} \mathrm{~cm}^{2} / \mathrm{W}$, which gives $\left|\chi^{(3)}\right|$ $\sim 10^{-12}$ esu.

In this paper, we used the ZS technique in the femtosecond ( $\mathrm{fs}$ ) and millisecond ( $\mathrm{ms}$ ) time scales to investigate the nonlinear properties of an IF (with uncoated particles), associated to electronic and thermal mechanisms, respectively. The nonlinear refractive indices are measured and the electric susceptibility is evaluated. The third-order nonlinear optical susceptibility of the ferrofluid is compared with those of other confined systems. The paper is organized as follows: in Section 2, both the femtosecond and millisecond formalisms used are presented; in Section 3, the details about the samples and the setups are given, followed by results and discussion in Section 4, and finally the conclusions in Section 5.

\section{THEORETICAL BACKGROUND}

\section{A. Femtosecond Time-Scale Regime}

The induced electric polarization of a medium with inversion symmetry, illuminated by a monochromatic Gaussian laser beam with electric field $\mathbf{E}$ and frequency $w$, can be written as: $P^{(3)}(w)=\varepsilon_{o} \mathrm{X}^{(3)}(w ; w,-w, w) E E^{*} E$, where $\varepsilon_{o}$ is the permittivity of free space. The nonlinear refractive index $n_{2}$ and the nonlinear absorption coefficient $\beta$, in a system whose response is local both in space and time, are defined as: $n(I)=n_{o}+n_{2} I$, and $\alpha(I)=\alpha_{o}+\beta I$, where $n(I)$ is the refractive index, $\alpha(I)$ is the absorption coefficient, and $I$ is the irradiance (or the intensity), $n_{o}$ and $\alpha_{o}$ are the linear index of refraction and absorption, respectively. It is important to notice that, in this time scale (femtoseconds), $n_{2}$ has an electronic origin. The real and imaginary parts of the third-order susceptibility are written as

$$
\begin{aligned}
& \operatorname{Re} \chi^{(3)}=2 n_{o}^{2} \varepsilon_{o} c n_{2}\left[\mathrm{~m}^{2} / \mathrm{V}^{2}\right], \\
& \operatorname{Im} \chi^{(3)}=n_{o}^{2} \varepsilon_{o} c^{2} \frac{\beta}{w}\left[\mathrm{~m}^{2} / \mathrm{V}^{2}\right],
\end{aligned}
$$

where $c$ is the velocity of light in vacuum and $w$ is the frequency of the light. In a ZS experiment, the far-field onaxis irradiance $I(z)$ is measured as a function of sample position $(z)$. For a sample where the nonlinear absorption is small, the on-axis normalized transmittance $\Gamma_{N}$ as function of the sample position $z$ is given approximately $\mathrm{by}^{2,11}$

$$
\Gamma_{N}(z) \simeq 1-\frac{\Delta \Phi_{o} 4 x}{\left(1+x^{2}\right)\left(9+x^{2}\right)}-\frac{\Theta 2\left(x^{2}+3\right)}{\left(1+x^{2}\right)\left(9+x^{2}\right)}
$$

where $\Delta \Phi_{o}$ is the on-axis nonlinear phase shift at focus, $x$ is the dimensionless sample position $x=z / z_{o}, \Theta=\beta I_{o} L_{\text {eff }}$, and $z_{o}=\pi \omega_{o}^{2} / \lambda$ is the Rayleigh range of the Gaussian beam with waist $\omega_{o}$. The nonlinear phase shift $\Delta \Phi_{o}$ is given by

$$
\Delta \Phi_{o}=-\frac{2 \pi n_{2} L_{\mathrm{eff}} I_{o}}{\lambda},
$$

where $\lambda$ is the wavelength, $L_{\text {eff }}=L\left(1-e^{-\alpha_{o} L}\right) / \alpha_{o}$ is the effective sample thickness, $L$ is the actual sample thickness, and $I_{o}$ is the on-axis intensity at the focus. The parameter $\Theta$ can be measured from the ZS experiments where all the transmitted light (not only the on-axis contribution) is measured by the detector. More details will be given in Section 3. In this case, Eq. (3) is written as

$$
\Gamma_{N}(z) \simeq 1-\frac{1}{2} \frac{\Theta}{1+x^{2}}
$$

\section{B. Millisecond Time-Scale Regime}

The nonlinear response of a colloidal medium in the millisecond time-scale regime is studied on the framework of the generalization of the thermal lens model. ${ }^{12,13}$ Let us briefly summarize the key points of this analysis. In the time-resolved version of the $\mathrm{ZS}$ experiment, a mechanical chopper provides a square-wave light intensity profile, which exhibits a periodical succession of on and off states of equal $\Delta t$ duration. During an on period, nonlinear effects appear, and temperature $T$ has enough time to reach a steady state in the sample. Conversely, during an off period, no nonlinear effects take place, and the temperature increase has time enough to decay. The sample position $z$ is fixed during a sequence of one on state and one off state. The experiment consists in measuring the sample transmitted intensity $[\Gamma(z, t)]$ as a function of time during the on period, at each (fixed) sample position $z$. The refractive index $n(r, t)$ of the colloidal medium depends on the colloidal particles volume-fraction $\varphi$, the temperature $T$, and the incident light intensity $I$. All these parameters are functions of the radial distance to the beam axis $r$ and the time $t$. The variation of the index of refraction $\delta n(r, t)$ can be expressed as the sum of terms arising from the temperature change $\delta T(r, t)$, the particle volume-fraction change $\delta \varphi(r, t)$, and light intensity $I(r, t)$ in the sample, due to the incident beam: $\delta n(r, t)=[\partial n / \partial T] \delta T(r, t)$ $+[\partial n / \partial \varphi] \delta \varphi(r, t)+[\partial n / \partial I] I(r, t)$. Assuming that in this time scale $\delta \varphi \rightarrow 0$, i.e., the Soret matter lens has no time to be formed, ${ }^{13}$ one has $\delta n(r, t)=[\partial n / \partial T] \delta T(r, t)+n_{2}^{\dagger} I(r, t)$, where $n_{2}^{\dagger}=\partial n / \partial I$. It is important to notice that in this time scale (milliseconds), $n_{2}^{\dagger}$ has a thermal origin. The on-axis optical transmittance $\Gamma(z, t)$ in the far field in a ZS experiment is written as ${ }^{12}$ 


$$
\Gamma(z, t)=\frac{\Gamma^{\prime}}{1-2 x\left[\frac{C_{N}}{\left(1+x^{2}\right)^{2}}+\frac{C_{T}}{1+x^{2}} \frac{t}{t+2 t_{c}}\right]+\left(1+x^{2}\right)\left[\frac{C_{N}}{\left(1+x^{2}\right)^{2}}+\frac{C_{T}}{1+x^{2}} \frac{t}{t+2 t_{c}}\right]^{2}},
$$

with $C_{N}=\left(8 P L z_{0} n_{2}^{\dagger}\right) /\left(\pi \omega_{0}^{4}\right)$ and $C_{T}=\left[(\partial n / \partial T)\left(L z_{0} \alpha_{0} P\right)\right] /$ $\left(\pi \omega_{o}^{2} \kappa\right)$, where $\Gamma^{\prime}$ is the sample transmitted intensity when the sample is at $|z| \gg z_{0}$, at any time $t \leqslant \Delta t, P$ and $\kappa$ are the incident power and the thermal conductivity of the sample, and $t_{c}=\left(\omega_{0}^{2}\left[1+x^{2}\right]\right) / 8 D$, with $D$ as the heat diffusivity of the sample. The normalized transmitted intensity $\Gamma_{N}(z)$ is defined in Eq. (7) and shows a typical peakvalley ZS curve behavior, with a peak-to-valley amplitude defined by Eq. (8):

$$
\begin{aligned}
\Gamma_{N}\left(z, t \rightarrow 0^{+}\right) & =\frac{\Gamma\left(z, t \rightarrow 0^{+}\right)}{\Gamma^{\prime}}=\Gamma_{N}(z), \\
\Delta \Gamma_{\mathrm{pv}}^{\text {theo }} & =\Gamma_{N}\left(z=z_{p}\right)-\Gamma_{N}\left(z=z_{v}\right),
\end{aligned}
$$

where $t \rightarrow 0^{+}$is the time corresponding to the first measured transmittance in an on period, $z_{p}$ and $z_{v}$ are the peak and valley positions in the ZS curve. This normalization is possible to be done since ferrofluids are strong absorbers, and the nonlinear effects are already present at $t \rightarrow 0^{+} .{ }^{14}$ In this framework, Eq. (7) may be written as

$$
\Gamma_{N}(z)=\frac{1}{1-2 x\left[\frac{C_{N}}{\left(1+x^{2}\right)^{2}}\right]+\left(1+x^{2}\right)\left[\frac{C_{N}}{\left(1+x^{2}\right)^{2}}\right]} .
$$

\section{EXPERIMENTAL}

\section{A. Samples}

The ferrofluid samples investigated are acid materials $(\mathrm{pH}=2)$, with $\mathrm{CoFe}_{2} \mathrm{O}_{4}$ magnetic particles positively charged, dispersed in water. They are labeled IF $i \quad(i=1$ $\rightarrow 4$ ), as a function of the particles diameter $\left(D_{r x}\right.$ in nanometers, obtained by x-ray experiments): IF $1 \Rightarrow D_{r x}$ $=11.6$; IF $2 \Rightarrow D_{r x}=8.8 ;$ IF $3 \Rightarrow D_{r x}=7.5$, and IF $4 \Rightarrow D_{r x}=4.5$. The ionic water-based ferrofluids elaboration is carried out in three fundamental steps ${ }^{15}$ : First, the ferrite nanoparticle synthesis, then the chemical surface treatment, and finally the peptization of the particles in a stable aqueous colloidal dispersion. $\mathrm{CoFe}_{2} \mathrm{O}_{4}$ oxide nanoparticles are prepared using hydrothermal coprecipitating aqueous solutions of a $\mathrm{Co}\left(\mathrm{NO}_{3}\right)_{2}-\mathrm{FeCl}_{3}$ mixture in an alkaline medium. ${ }^{16}$ After the coprecipitation step, the precipitate is washed in order to suppress the high ionic strength of the medium, and the particle surface is cleaned by a $\left(2 \mathrm{~mol} \mathrm{~L}^{-1}\right) \mathrm{HNO}_{3}$ solution. Moreover, to obtain the thermodynamical stability of the particles, an empirical process is used: the precipitates are boiled with a $0.5 \mathrm{~mol} \mathrm{~L}-1$ $\mathrm{Fe}\left(\mathrm{NO}_{3}\right)_{3}$ solution. Then, the particles are conveniently peptized in an acidic medium by adjustment of the ionic strength, resulting in a stable sol of high quality. The crystalline structure and the mean size of our magnetic nanoparticles were carried out from x-ray diffraction measurements performed on powder samples, at $25^{\circ} \mathrm{C}$, obtained after evaporation of the liquid carrier. The chemical composition of the synthesized nanoparticles was checked: the concentrations of $\mathrm{Fe}$ and Co ions were determined by inductively coupled plasma atomic emission spectroscopy (ICP-AES). Then, the volume fractions of the magnetic material $\varphi$ of all precursor samples were deduced. In the following experiments, the precursor dispersions were diluted in order to obtain samples whose volume fractions are sufficiently low $(\varphi \sim 1 \%)$ to neglect the magnetic dipolar interaction between particles. In the ZS experiments, all the samples are encapsulated between optical glass plates with dimensions $(20 \mathrm{~mm}$ $\times 10 \mathrm{~mm} \times 1 \mathrm{~mm}$ ), with a Teflon spacer $L=24 \mu \mathrm{m}$ thick. The values of $\varphi$ are chosen by analyzing the transmittances and the sample response to the incident laser beam. $\varphi_{\min }$ is the minimum volume fraction of the magnetic material, that gives a good signal-to-noise ratio in the ZS experiment, with the maximum available beam power. $\varphi_{\max }$ is the maximum volume fraction of magnetic material that does not show thermal-induced hydrodynamic instabilities in the sample due to the laser heating, at the lowest value of the beam power. So, we chose $\varphi_{\min }$ $<\varphi<\varphi_{\max }$. All the measurements were performed at $T$ $=20^{\circ} \mathrm{C}$.

\section{B. Linear Optical Absorption and Linear Refractive Index}

The linear optical absorption $\left(\alpha_{o}\right)$ of the samples is measured with a UV-2800 single beam scanning UV-Visible spectrophotometer (at $\lambda_{s}=532 \mathrm{~nm}$ ). The linear refractive index $\left(n_{o}\right)$ of the different samples (at $\lambda_{r}=589 \mathrm{~nm}$ ) is measured using an Abbe refractometer (Carl Zeiss) with a temperature controlled device (thermal bath Brookfield TC 500 with $0.01{ }^{\circ} \mathrm{C}$ accuracy).

\section{Z-Scan Apparatus and Technique}

1. Femtosecond Time-Scale Setup

A mode-locked Ti:sapphire laser $(\lambda=790 \mathrm{~nm})$, pumped by a $5 \mathrm{~W}$ cw doubled frequency $\mathrm{Nd}$ : $\mathrm{YVO}_{4}\left(\lambda_{p}=532 \mathrm{~nm}\right)$ laser, is used in these ZS experiments. The pulse width is of $508.5 \mathrm{fs}$, and the irradiance on the sample ranges from 0.16 to $0.22 \mathrm{GW} / \mathrm{cm}^{2}$. By using a pulse selector, the femtosecond pulses are separated (peak-to-peak) in time by $240 \mathrm{~ns}$ (originally, without the pulse selector, the Ti:sapphire pulse frequency is $82 \mathrm{MHz}$ ). A lens of focal distance $f=88.3 \mathrm{~mm}$, which provides a minimum waist of $\omega_{o}$ $=27.3 \mu \mathrm{m}$, with a Rayleigh length $z_{o}=2.97 \mathrm{~mm}$, is placed before the sample. The sample is put in the beam path and is moved around $z=0$, before and after the focal point, along the $z$ axis. The transmitted beam goes to the detector and is recorded by an oscilloscope. The beam intensity $I$ at a point $z$ changes due to a change of the beam waist $[\omega(z)]$. This intensity $I$ reaches the maximum value at the focal point, then it decreases for far points. The optical 
nonlinear characteristics of the sample arise mainly near $z=0$ due to high intensity $I$, and at far points, it is not noticeable. For the measurement of the nonlinear refractive index only the on-axis transmittance is measured. On the other hand, to measure the nonlinear absorption, a converging lens is placed after the sample to collect all the transmitted beam at the detector. The incident (peak) laser power used in each experiment is $I_{o} \sim 10^{12} \mathrm{~W} / \mathrm{m}^{2}$.

\section{Millisecond Time-Scale Setup}

In the time-resolved version of the ZS experiment, a mechanical chopper modulates the laser beam (Gaussian profile) providing a square-wave pulse, which exhibits a periodical succession of on and off states of equal $\Delta t$ duration. The sample position $z$ is fixed during a sequence of one on state and one off state. The experiment consists of measuring the sample transmitted intensity $(\Gamma(z, t))$ as a function of time during the on period at each (fixed) sample position $z$. A cw doubled frequency $\mathrm{Nd}: \mathrm{YVO}_{4}(\lambda$ $=532 \mathrm{~nm}$ ) laser is used in the ZS measurements. The laser beam is chopped (at $18 \mathrm{~Hz}$ ) or controlled by a shutter for larger time intervals $\Delta t$ and focused by a lens (focal distance $f=100 \mathrm{~mm}$ ), which provides a minimum waist of $\omega_{o}=20.3 \mu \mathrm{m}$, with a Rayleigh length $z_{o}=2.43 \mathrm{~mm}$. The time resolution in the experiment is $\sim 100 \mu \mathrm{s}$, and the ZS step used is $\Delta z=0.5 \mathrm{~mm}$. For each ZS measurement, the sample is placed in a chariot, which moves it along the $z$ axis of the laboratory frame, with its largest surface perpendicular to the laser beam. Depending on the magnetic particle concentration, different incident beam powers are used in the experiments. A sketch of the experimental setup can be found in Ref. 12 .

\section{RESULTS AND DISCUSSION}

\section{A. Femtosecond Time-Scale Processes}

Figure 1 shows typical on-axis [Fig. 1(a)] and total [Fig. 1(b)] ZS transmittances as a function of $z$ of the ionic IF2 magnetic colloid. Each point represents the average of five independent measurements. The solid curves represent the best fits of Eqs. (3) and (5), respectively, to the experimental data. From these fittings, the parameters $n_{2}$ and $\beta$ can be obtained. Their values for the set of samples investigated are given in Table 1 . The order of magnitude of $n_{2}$ obtained in our experiment $\left(10^{-11} \mathrm{~cm}^{2} / \mathrm{W}\right)$ clearly indicates that the mechanism responsible for this response is not thermal. ${ }^{1}$ This order of magnitude is ten times smaller than that found in chitosan/CdSe-ZnS quantum dot (QD) core-shell multilayer films (at the same wavelength), where the typical diameters of the CdS and ZnS QDs were $\sim 4 \mathrm{~nm}$, and the film thickness per bilayer $\sim 8 \mathrm{~nm}^{17}$

With our measured values of $n_{2}$ and $n_{o}$ $(=1.328 \pm 0.002)$, it is possible to calculate the real part of the third-order nonlinear electric susceptibility of the samples using Eq. (1). These values are presented in Table 1 . To check if we are really probing $\operatorname{Re} \chi^{(3)}$ and not high-order susceptibilities, we performed the ZS experiment with three different incident (peak) laser powers: $I_{o}=3.87,4.04$, and $4.71\left(\times 10^{11} \mathrm{~W} / \mathrm{m}^{2}\right)$. The variation in the values of $n_{2}$ obtained was smaller than $3 \%$ indicating that our ZS experiment measures $\operatorname{Re} \chi^{(3)}$ and not high-
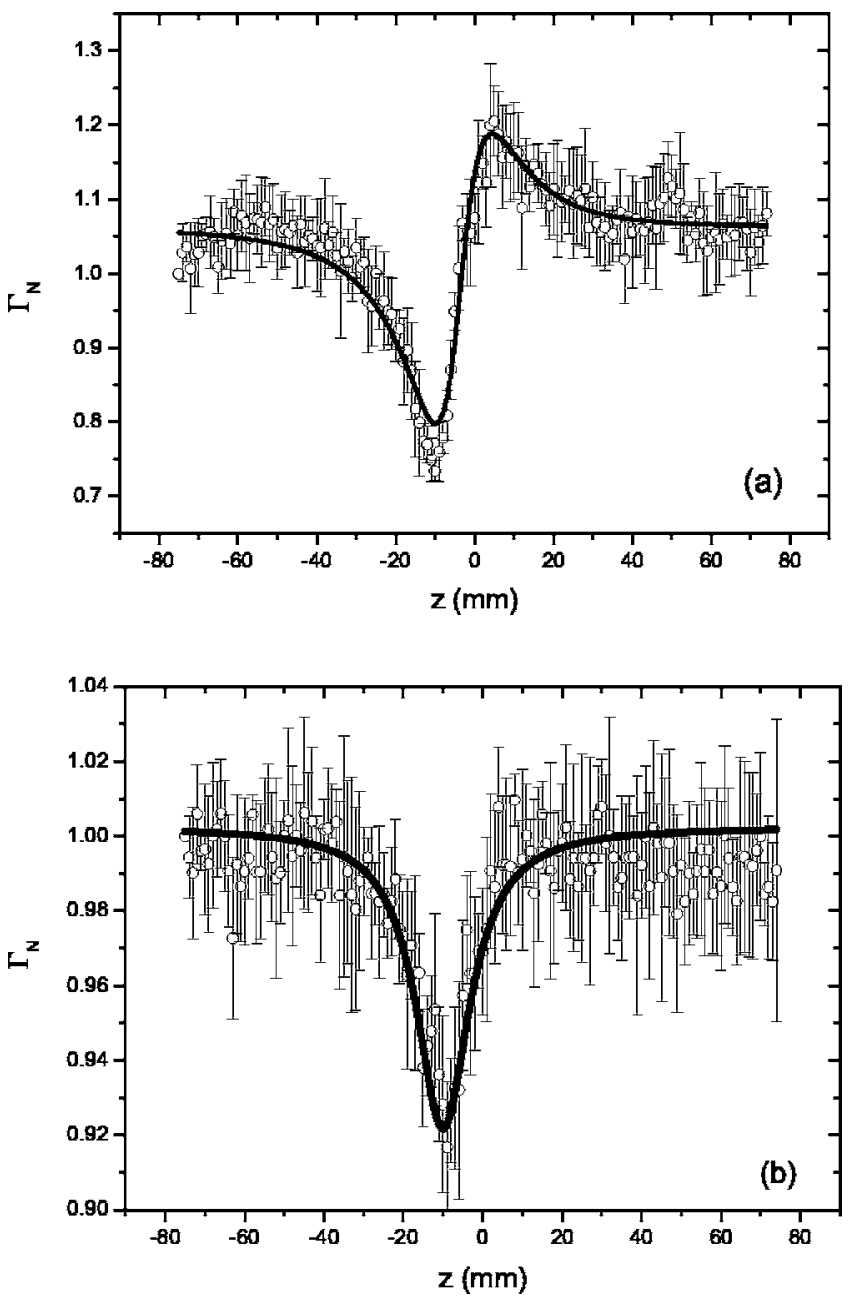

Fig. 1. Typical ZS curves of the normalized transmittances in the femtosecond experiment, as a function of $z$. Water-based ionic sample IF2: (a) on-axis transmittance. The solid curve represents the best fit of Eq. (3) to the experimental data (b) Total transmittance. The solid curve represents the best fit of Eq. (5) to the experimental data.

Table 1. Femtosecond ZS Experiment Results ${ }^{a}$

\begin{tabular}{ccccc}
\hline Label & $\begin{array}{c}n_{2} \\
\left(10^{-11} \mathrm{~cm}^{2} / \mathrm{W}\right)\end{array}$ & $\begin{array}{c}\beta \\
\left(10^{3} \mathrm{~cm} / \mathrm{GW}\right)\end{array}$ & $\begin{array}{c}\operatorname{Re} \chi^{(3)} \\
\left(10^{-17} \mathrm{~m}^{2} / \mathrm{V}^{2}\right)\end{array}$ & $\begin{array}{c}\operatorname{Im} \chi^{(3)} \\
\left(10^{-18} \mathrm{~m}^{2} / \mathrm{V}^{2}\right)\end{array}$ \\
\hline IF1 & $2.1 \pm 0.2$ & $2.7 \pm 0.1$ & $2.0 \pm 0.2$ & $1.6 \pm 0.4$ \\
IF2 & $2.4 \pm 0.1$ & $3.22 \pm 0.06$ & $2.2 \pm 0.1$ & $1.9 \pm 0.5$ \\
IF3 & $2.7 \pm 0.2$ & $5.3 \pm 0.6$ & $2.6 \pm 0.2$ & $3.1 \pm 0.9$ \\
IF4 & $3.5 \pm 0.3$ & $9.0 \pm 0.5$ & $3.2 \pm 0.3$ & $5 \pm 1$ \\
\hline
\end{tabular}

${ }^{a}$ Label of the investigated magnetic colloidal samples, nonlinear index of refraction $\left(n_{2}\right)$, nonlinear optical absorption $(\beta)$, and real and imaginary parts of $\chi^{(3)}$.

order susceptibilities. The sign of $n_{2}$ is positive, indicating a convergent-lens-type behavior at this time-scale regime.

The order of magnitude of $\beta$ found in our experiments is $\sim 10^{3} \mathrm{~cm} / \mathrm{GW}$. This order of magnitude is also the same found in chitosan/CdSe-ZnS core-shell QD multilayer films. The value of $\beta$ for this multilayer film is much larger than that in the bulk CdSe $(\sim 6.4 \mathrm{~cm} / \mathrm{GW})$. As likely as in the case of the multilayer QDs, confining effects in our nanoparticles seem to be responsible for this order of magnitude of $\beta$ found in our samples. We will 
come back to this point in the following sentences. Different from the case of $n_{2}$, the same experiment performed at different values of $I_{o}$ shows a variation of about $30 \%$ in the value of $\beta$, in the range of intensities investigated (Fig. 2). This fact indicates that, besides $\chi^{(3)}$, high-order susceptibilities have been probed in our ZS experiment to determine $\beta$. However, we can evaluate the order of magnitude of the imaginary part of $\chi^{(3)}$ using Eq. (2) and $w$ $=2.386 \times 10^{15} \mathrm{~Hz}$, being $\operatorname{Im} \chi^{(3)} \sim 10^{-18} \mathrm{~m}^{2} / \mathrm{V}^{2}$. This problem, in the determination of the $\operatorname{Im} \chi^{(3)}$ by using the ZS technique and the procedure described here, is expected because when the total transmitted intensity is collected by the lens and measured by the detector, not only may we collect the contribution from the third-order susceptibility, but also we may collect the high-order contributions (if present). This fact limits the application of the ZS technique for this particular measurement. ${ }^{18}$

The modulus of $\chi^{(3)}$, evaluated from our data, is shown in Fig. 3, being of the order of $10^{-17} \mathrm{~m}^{2} / \mathrm{V}^{2}$ (or, $10^{-9} \mathrm{esu}$ ): the smaller the magnetic particle diameter, the bigger the modulus of $\chi^{(3)}$. It is interesting to compare these values with those of other confined systems. In the case of the sol-gel derived $\alpha-\mathrm{Fe}_{2} \mathrm{O}_{3}, \gamma-\mathrm{Fe}_{2} \mathrm{O}_{3}$, and $\mathrm{Fe}_{3} \mathrm{O}_{4}$ thin films (a two-dimensional-type confined system), $\chi^{(3)}$ are 5.8 $\times 10^{-11} \mathrm{esu}, \quad 2.1 \times 10^{-11} \mathrm{esu}, \quad$ and $4 \times 10^{-10} \mathrm{esu}$, respectively. ${ }^{8}$ These are the highest values among inorganic oxides. In the case of the $\alpha-\mathrm{Fe}_{2} \mathrm{O}_{3}$ and $\gamma-\mathrm{Fe}_{2} \mathrm{O}_{3}$, these high values were attributed to the pair excitation process while, in the case of the $\mathrm{Fe}_{3} \mathrm{O}_{4}$, it was attributed to both one-photon and three-photon resonances. $\mathrm{Fe}_{2} \mathrm{O}_{3}$-coated nanoparticles dispersed in toluene and hydrosol ${ }^{10}$ presented $\left|\chi^{(3)}\right| \sim 10^{-12}$ esu. In our IF colloidal system, the magnitude of $\chi^{(3)}$ is 2 orders of magnitude bigger than that of the $\alpha-\mathrm{Fe}_{2} \mathrm{O}_{3}$ and $\gamma-\mathrm{Fe}_{2} \mathrm{O}_{3}$ and, in the case of the $\mathrm{Fe}_{3} \mathrm{O}_{4}, 1$ order of magnitude bigger. A possible origin of the high values of $\chi^{(3)}$ found in IF may be confining effects related to the physical nanoscale of the magnetic particles. This hypothesis is reinforced by the dependence of $\chi^{(3)}$ with the particle's diameter (Fig. 3). The IF shows the highest $\left|\chi^{(3)}\right|$ among other two-dimensional (thin films) and dot-type (coated nanoparticles) confined sys-

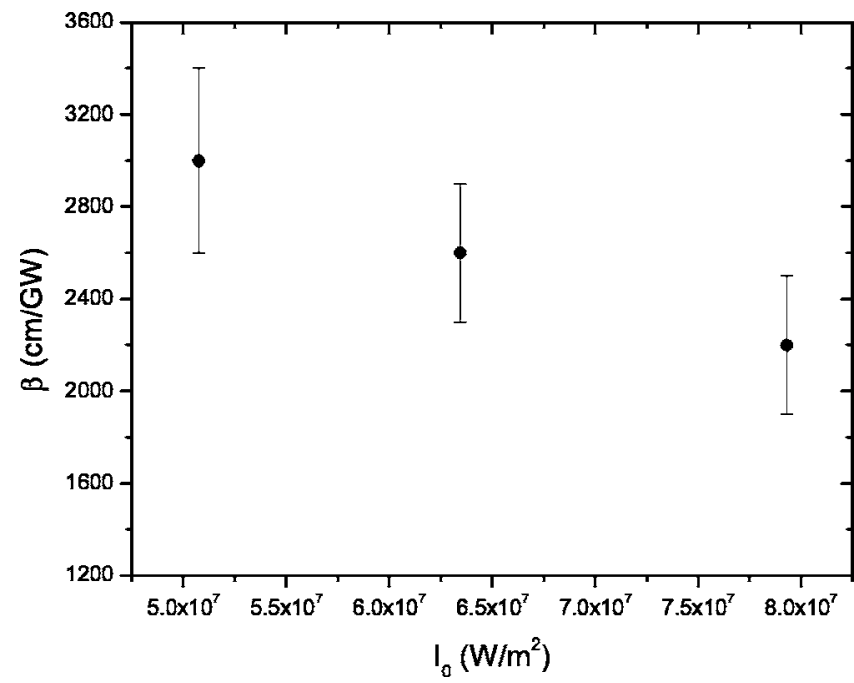

Fig. 2. Nonlinear optical absorption $\beta$ as a function of the incident (peak) laser power $I_{o}$. Water-based ionic sample IF2.

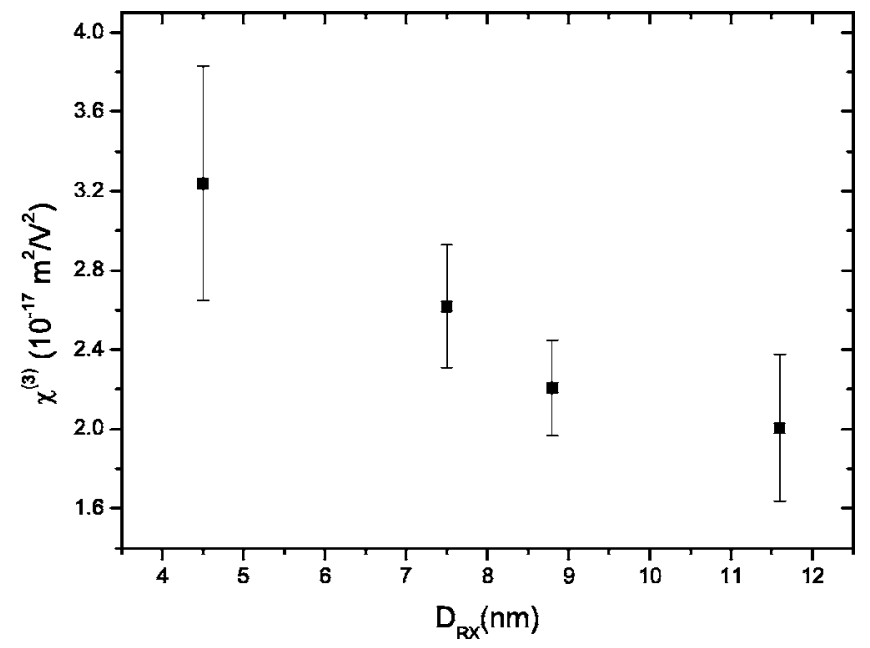

Fig. 3. Modulus of $\chi^{(3)}$ as a function of the magnetic particle's diameter. Water-based ionic sample IF2.

Table 2. Millisecond ZS Experiment Results ${ }^{a}$

\begin{tabular}{ccr}
\hline Label & $-n_{2}^{\dagger}\left(10^{-7} \mathrm{~cm}^{2} / \mathrm{W}\right)$ & \multicolumn{1}{c}{$\alpha_{o}\left(\mathrm{~cm}^{-1}\right)$} \\
\hline IF1 & $2.76 \pm 0.14$ & $205.75 \pm 0.32$ \\
IF2 & $2.30 \pm 0.11$ & $201.01 \pm 0.45$ \\
IF3 & $1.80 \pm 0.09$ & $196.07 \pm 0.51$ \\
IF4 & $1.46 \pm 0.07$ & $98.15 \pm 0.11$ \\
\hline
\end{tabular}

${ }^{a}$ Label of the investigated magnetic colloidal samples, nonlinear index of refraction $\left(n_{2}^{\dagger}\right)$, and linear light absorption coefficient at $532 \mathrm{~nm}\left(\alpha_{o}\right)$.

tems. Different from the case of the $\mathrm{Fe}_{2} \mathrm{O}_{3}$-coated nanoparticles, our particles are uncoated but are electrically charged.

\section{B. Millisecond Time-Scale Processes}

As pointed out at the end of Section 2, the data treatment proposed at this time scale is adequate to strong absorbers. To check the hypothesis that ferrofluids fulfill this condition, the linear light absorption coefficient $\left(\alpha_{o}\right)$ of the different samples is measured, and the values are given in Table 2. These values (of the order of $10^{2} \mathrm{~cm}^{-1}$ ) support the theoretical framework used to analyze the ZS curves following the normalization procedure present in Eq. (7).

Figure 4 shows typical on-axis ZS transmittance as a function of $z$ of the ionic IF2 magnetic colloid. Each point represents the average of five independent measurements. The data treatment to obtain the values of $n_{2}^{\dagger}$ from the ZS curves is described in detail in Refs. 13 and 14. The dimensionless parameter $C_{N}$ is obtained by means of a master curve, and knowing $P, L, z_{o}$, and $\omega_{o}$, we can calculate $n_{2}^{\dagger}$. Within our accuracy, any nonlinear absorption is found in all the IF samples investigated (data not shown), in this time-scale regime.

Different, from the femtosecond time-scale regime, $n_{2}^{\dagger}$ $<0$, its order of magnitude is $10^{-7} \mathrm{~cm}^{2} / \mathrm{W}$, which is characteristic of a thermal mechanism. $n_{2}^{\dagger}$ depends on the magnetic particle's diameter: the smaller the $D_{r x}$, the smaller the absolute value of $n_{2}^{\dagger}$ (Fig. 5). The sign of $n_{2}^{\dagger}$ is negative, indicating a divergent-lens-type behavior at this time-scale regime. Since the magnetic particles are strong absorbers at this wavelength, it is expected that they in- 
crease their temperature due to the laser-light absorption and transfer this heat to the fluid carrier, in this case, the water. This mechanism increases locally the sample temperature imposing a modification of its local density, originating the divergent-lens-type effect. In this sense, the larger surface area of the larger particles favors the heat conduction from them to the carrier fluid (i.e., the water), which is responsible for the local density modifications of the sample under the laser illumination.

\section{CONCLUSIONS}

The Z-scan technique is used to measure the nonlinear refractive index and the nonlinear absorption coefficient of ionic magnetic colloids as a function of the nanoparticles' diameter in the femto- and millisecond time-scale regimes. In the femtosecond regime, the magnetic colloids show a convergent-lens-type behavior, and, at this time scale, the processes responsible for the systems' optical response are expected to be from electronic origin. Both $n_{2}$ and $\beta$ were shown to depend on the particles' diameter: the smaller the particle's diameter, the bigger $n_{2}$ and $\beta$.

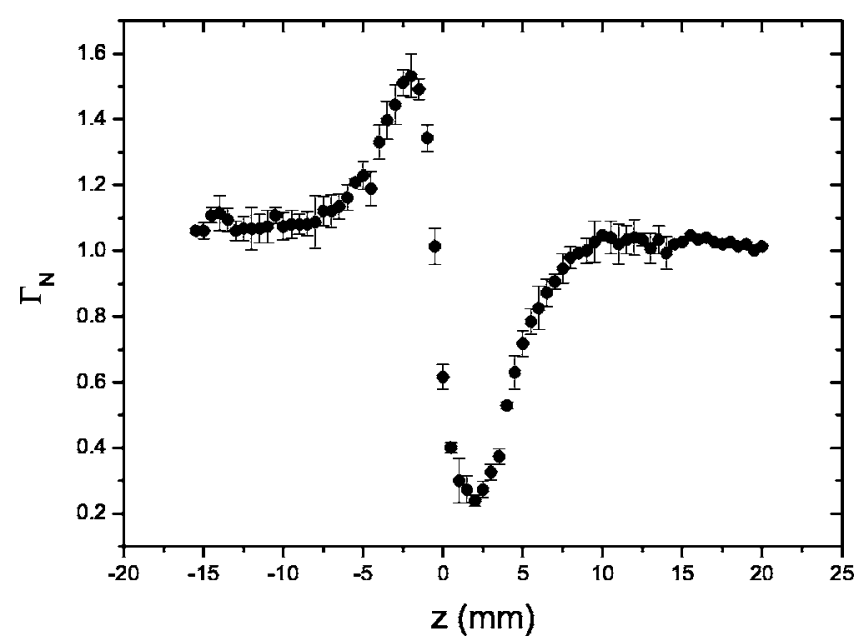

Fig. 4. Typical ZS curve of the normalized transmittances in the millisecond experiment, as a function of $z$. Water-based ionic sample IF2.

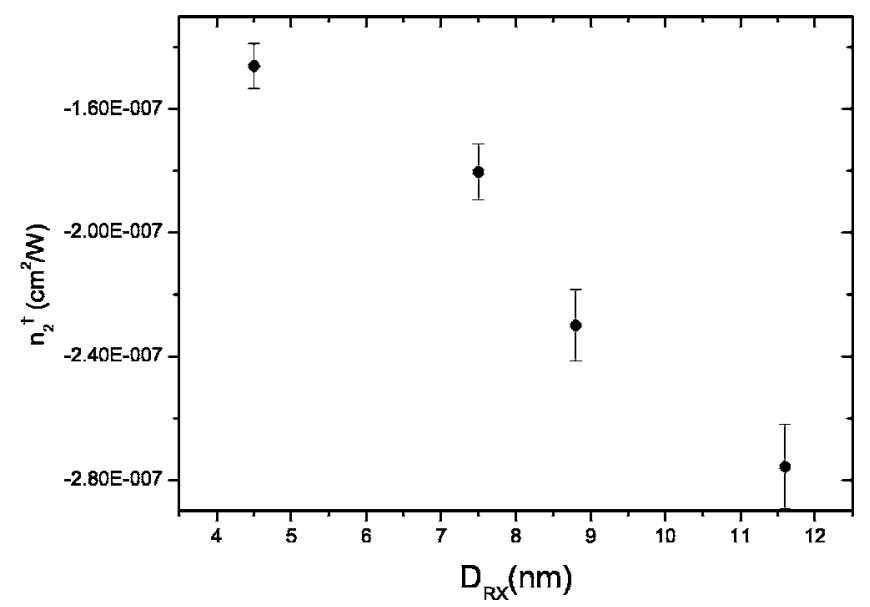

Fig. 5. Nonlinear refraction index $n_{2}^{\dagger}$ as a function of the magnetic particle's diameter $D_{r x}$.
From these values, the third-order susceptibility $\chi^{(3)}$ could be calculated and showed the same type of behavior with respect to the magnetic nanoparticles' diameter. A possible origin of the high values of $\chi^{(3)}$ found in ionic ferrofluids may be confining effects related to the physical nanoscale of the magnetic particles. The ionic ferrofluid shows the highest $\left|\chi^{(3)}\right|$ among other two-dimensional (thin films) and dot-type (coated nanoparticles) confined systems. When the systems are investigated in the millisecond time-scale regime, the magnetic colloids show a divergent-lens-type behavior, and $\left|n_{2}^{\dagger}\right|$ is smaller for systems with the smallest nanoparticle diameter. At this time scale, the processes responsible for the systems' responses are from thermal origin.

\section{ACKNOWLEDGMENTS}

We are in debt to FAPESP (Fundação de Amparo à Pesquisa do Estado de São Paulo), CAPES (Fundação Coordenação Aperfeiçoamento de Pessoal de Nível Superior), and $\mathrm{CNPq}$ (Instituto do Milênio de Fluidos ComplexosIMFCx) for financial support. F. L. S. Cuppo's help in the experimental setup is acknowledged.

A. M. Figueiredo's e-mail address is afigueiredo@if.usp.br.

\section{REFERENCES}

1. R. W. Boyd, Nonlinear Optics (Academic, 1992).

2. M. Sheik-Bahae, A. A. Said, and E. W. Van Stryland, "High sensitivity single-beam $n_{2}$ measurements," Opt. Lett. 14, 955-957 (1989).

3. R. E. Rosensweig, Ferrohydrodynamics (Cambridge U. Press, 1985).

4. R. Massart, "Preparation of aqueous magnetic liquids in alkaline and acid media," IEEE Trans. Magn. 17, 1247-1248 (1981).

5. A. M. Figueiredo Neto and S. R. A. Salinas, The Physics of Lyotropic Liquid Crystals: Phase Transitions and Structural Properties (Oxford U. Press, 2005).

6. J. W. M. Bulte, "Magnetic nanoparticles as markers for cellular MR imaging," J. Magn. Magn. Mater. 289, 423-427 (2005).

7. T. Hashimoto, T. Yoko, and S. Sakka, "Third-order nonlinear optical susceptibility of $\alpha$-iron (III) oxide thin film prepared by the sol-gel method," J. Ceram. Soc. Jpn. 101, 64-68 (1993).

8. T. Hashimoto, T. Yamada, and T. Yoko, "Third-order nonlinear optical properties of sol-gel derived $\alpha-\mathrm{Fe}_{2} \mathrm{O}_{3}, \gamma-$ $\mathrm{Fe}_{2} \mathrm{O}_{3}$, and $\mathrm{Fe}_{3} \mathrm{O}_{4}$ thin films," J. Appl. Phys. 80, 3184-3190 (1996).

9. C. C. Wang, "Empirical relation between the linear and the third-order nonlinear optical susceptibility," Phys. Rev. B 2, 2045-2048 (1970).

10. B. Yu, C. Zhu, F. Gan, X. Wu, G. Zhang, G. Tang, and W. Chen, "Optical nonlinearities of $\mathrm{Fe}_{2} \mathrm{O}_{3}$ nanoparticles investigated by Z-scan technique," Opt. Mater. 8, 249-254 (1997).

11. H. J. Yuan, L. Li, and P. Palffy-Muhoray, "Nonlinear birefringence of nematic liquid crystals," Proc. SPIE 1307, 363-373 (1990).

12. S. Alves, A. Bourdon, and A. M. Figueiredo Neto, "Generalization of the thermal lens model formalism to account for thermodiffusion in a single-beam Z-scan 
experiment: determination of the Soret coefficient," J. Opt. Soc. Am. B 20, 713-718 (2003).

13. S. Alves, F. L. S. Cuppo, and A. M. Figueiredo Neto, "Determination of the nonlinear refractive index of lyotropic mixtures with and without the ferrofluid doping: a time-resolved Z-scan experiment in millisecond time scales," J. Opt. Soc. Am. B 23, 67-74 (2006).

14. S. Alves, A. Bourdon, and A. M. Figueiredo Neto, "Investigation of the Soret coefficient in magnetic fluids using the Z-scan technique," J. Magn. Magn. Mater. 289 285-288 (2005).

15. M. H. Sousa, J. Depeyrot, G. J. da Silva, M. C. F. L. Lara, and F. A. Tourinho, "New electric double-layered magnetic fluids based on copper, nickel, and zinc ferrite nanostructures," J. Phys. Chem. B 105, 1168-1175 (2001).

16. F. A. Tourinho, R. Franck, and R. Massart, "Aqueous ferrofluids based on manganese and cobalt ferrites," J. Mater. Sci. 25, 3249-3254 (1990).

17. X. Wang, Y. Du, S. Ding, Q. Wang, G. Xiong, M. Xie, X. Shen, and D. Pang, "Preparation of third-order optical nonlinearities of self-assembled chitosan/CdSe-ZnS coreshell quantum dots multilayer films," J. Phys. Chem. B 110, 1566-1570 (2006).

18. F. Yoshino, S. Polyakov, M. Liu, and G. Stegeman, "Observation of three-photon enhanced four-photon absorption," Phys. Rev. Lett. 91, 0639021 (2003). 